\title{
FibroScan Detection of Fatty Liver/Liver Fibrosis in 2266 Cases of Chronic Hepatitis B
}

\author{
Tingshan $\mathrm{He}^{1}$, Jing $\mathrm{Li}^{1}$, Yanling Ouyang ${ }^{1}$, Guotao $\mathrm{Lv}^{1}$, Xiaofeng Ceng ${ }^{2}$, Zhiqiao Zhang ${ }^{1 *}$ \\ and Jianqiang Ding ${ }^{1 *}$
}

${ }^{1}$ Department of Infectious Diseases, Shunde Hospital, Southern Medical University, Shunde, Guangdong, China; ${ }^{2}$ Department of Pathology, Shunde Hospital, Southern Medical University, Shunde, Guangdong, China

\begin{abstract}
Background and Aims: FibroScan is used to determine liver stiffness and controlled attenuation parameter (referred to as CAP) scores in patients, including those with chronic hepatitis $\mathrm{B}(\mathrm{CHB})$. We used FibroScan to detect the incidence of fatty liver and fibrosis in CHB patients, and to assess the correlation of FibroScan measurements with blood chemistry tests. Methods: CHB patients enrolled in this study were divided independently for three separate analyses (of fibrosis, cirrhosis, and fatty liver) based on FibroScan results. Basic information, blood chemistry test results, liver fibrosis parameters, and FibroScan results were collected. T-tests and Pearson's analyses were used to analyze the correlations between FibroScan liver stiffness measurement/CAP values and liver function, blood fat, uric acid metabolite, fibrosis, and hepatitis B virus load. Results: A total of 2266 CHB patients were enrolled in the study and divided into three groups: non-significant and significant fibrosis; non-cirrhosis and early cirrhosis; and non-fatty and fatty liver. Spearman's statistical analyses showed that liver stiffness measurement or CAP values correlated with sex $(r=0.137)$, age $(r=0.119)$, glutamic-pyruvic transaminase $(r=0.082)$, glutamic-oxaloacetic transaminase $(r=-0.172)$, gamma-glutamyltransferase $(r=0.225)$, albumin $(r=0.150)$, globulin $(r=-0.107)$, total bilirubin $(r=-0.132)$, direct bilirubin $(r=-0.145)$, white blood cell count $(r=0.254)$, hemoglobin $(r=0.205)$, platelets $(r=0.206)$, total cholesterol $(r=0.214)$, high density lipoprotein $(r=-0.243)$, low density lipoprotein $(r=0.255)$, apolipoprotein B $(r=0.217)$, hyaluronic acid $(r=-0.069)$, laminin $(r=-0.188)$, procollagen type IV $(r=-0.067)$ and hepatitis B viral DNA load $(r=-0.216)$. Conclusions: FibroScan is a non-invasive device that can detect the occurrence of fatty liver or liver fibrosis in CHB patients.
\end{abstract}

Keywords: FibroScan; Chronic hepatitis B; Fatty liver; Liver fibrosis; Cirrhosis. Abbreviations: Alb, albumin; ALD, alcoholic fatty liver disease; ALT, glutamicpyruvic transaminase; AST, glutamic-oxaloacetic transaminase; CAP, controlled attenuation parameter; $\mathrm{CHB}$, chronic hepatitis $\mathrm{B}$; $\mathrm{DB}$, direct bilirubin; $\mathrm{EC}$, early cirrhosis; FL, fatty liver; GGT, gamma-glutamyltransferase; Hb, hemoglobin; $H D L$, high density lipoprotein; LDL, low density lipoprotein; LSM, liver stiffness measurement; NAFLD, non-alcoholic fatty liver disease; NC, non-cirrhosis; NFL, nonfatty liver; NSF, non-significant fibrosis group; Pl, platelet; SF, significant fibrosis; $\mathrm{TB}$, total bilirubin; TE, transient elastography; WBC, white blood cell.

Received: 14 October 2019; Revised: 18 March 2020; Accepted: 31 March 2020 *Correspondence to: Jianqiang Ding, Department of Infectious Diseases, Shunde Hospital, Southern Medical University, $1^{\#}$ Jiazi Road, Shunde, Guangdong 528308, China. Tel: +86-15218853076, E-mail: jding18@foxmail.com; Zhiqiao Zhang, Department of Infectious Diseases, Shunde Hospital, Southern Medical University, $1^{\#}$ Jiazi Rd, Shunde, Guangdong 528308, China. Tel: +8615876129625, E-mail: sdgrxjbk@163.com
Citation of this article: He T, Li J, Ouyang Y, Lv G, Ceng X, Zhang $Z$, et al. FibroScan detection of fatty liver/liver fibrosis in 2266 cases of chronic hepatitis B. J Clin Transl Hepatol 2020;8(2):113-119. doi: 10.14218/JCTH.2019.00053.

\section{Introduction}

The liver is the largest digestive organ in the human body and participates in the metabolism of most substances. Therefore, liver damage, which can occur due to a variety of reasons, impacts a large proportion of the bodily metabolism, including metabolization of blood lipids, blood sugar, uric acid, and proteins. ${ }^{1}$ Fatty liver disease refers to steatosis, in which the weight of liver fat accounts for more than $5 \%$ of the total liver weight, or where the histological appearance of fat accounts for $30 \%$ or more of liver volume. ${ }^{2}$ Fatty liver includes alcoholic fatty liver disease (ALD) and non-alcoholic fatty liver disease (NAFLD). Recently, NAFLD has become a particular area of interest in research, as this disease is increasingly seen in the clinic, but it could be improved through relatively simple and cost-effect changes in lifestyle. ${ }^{3}$

Studies have found that chronic hepatitis $\mathrm{B}(\mathrm{CHB})$ is a critical cause of fatty liver. As there is a high incidence of $\mathrm{CHB}$ in China, it is critical to determine whether $\mathrm{CHB}$ is associated with either liver fibrosis or fatty liver in these patients. ${ }^{4,5}$ Recently, a novel non-invasive technique called transient elastography (TE) was developed to assess liver fibrosis/ fatty liver. This technique induces a shear wave in the liver and measures the velocity of the wave in real time. Based on this technique, the FibroScan 502 device was developed in 2001 by Echosens (Paris, France), and has since been used in hospitals. ${ }^{6}$ Using FibroScan, values for liver stiffness measurement(LSM) and controlled attenuation parameter(CAP), indicating liver fibrosis and fatty liver respectively, can now be obtained non-invasively and in real-time. Therefore, FibroScan is an advanced non-invasive quantitative detection device for liver hardness and steatosis, which greatly improves early detection rates of these injuries. FibroScan is, thus, suitable for the diagnosis of fatty liver, liver fibrosis, and cirrhosis, which commonly exist alongside chronic viral hepatitis, alcoholic hepatitis, autoimmune liver diseases, and other liver conditions. ${ }^{7-9}$

In this clinical study, we collected data and information from 2266 CHB patients to assess the value of using FibroScan as a non-invasive tool for the diagnosis of fatty liver/ liver fibrosis, and to determine the correlation between FibroScan results and blood chemistry test results. 
He T. et al: FibroScan for $\mathrm{CHB}$

Methods

\section{Study design and patients}

A total of 2266 patients with CHB were enrolled in this study between December 2014 and August 2018 in the Department of Infectious Diseases, Shunde Hospital, Southern Medical University. These patients were divided into three different groups according to their FibroScan results, combined with measured clinical manifestations. Group 1 was divided by the degree of fibrosis. Group 2 was stratified by the level of cirrhosis. Group 3 was separated based on the absence (3a) or presence $(3 \mathrm{~b})$ of fatty liver.

\section{Blood chemical tests}

Blood biochemical indicators (lipids, glucose, liver function, fibrosis) were quantified using an Olympus Au1000 automatic biochemical analyzer (Shanghai Kehua - Dongling Diagnostic Products Co., Ltd., Shanghai, China).

\section{HBV DNA load in sera}

Blood HBV DNA load was measured with a quantitative realtime PCR kit (DA AN Gene, Guangzhou, China) using the LightCycler 96 system (Roche Molecular Systems, Basel, Switzerland). ${ }^{10}$

\section{LSM and CAP values detected by FibroScan}

We quantified LSM and CAP values using FibroScan 502 to assess the degree of fatty liver or liver fibrosis in CHB patients. According to the Operator's instructions, patients with CAP scores $\geq 237.7 \mathrm{db} / \mathrm{m}$ were defined as fatty liver patients, in which $237.7-259.3 \mathrm{db} / \mathrm{m}$ was defined as mild fatty liver, $259.4-292.3 \mathrm{db} / \mathrm{m}$ was defined as moderate fatty liver, and $>292.3 \mathrm{db} / \mathrm{m}$ was defined as severe fatty liver. LCM values of $7 \mathrm{kPa}, 9.5 \mathrm{kPa}$, and $12.5 \mathrm{kPa}$ demarcated the boundaries between no significant fibrosis (S1 of the METAVIR classification system), significant fibrosis (S2), severe fibrosis (S3), and cirrhosis (S4). ${ }^{11}$

\section{Liver tissues}

Liver biopsy samples were obtained from CHB in-patients. Human healthy liver tissue samples were provided by Xi'an Alenabio Inc. (Xi'an, China) and used as the negative control.

\section{Sirius Red staining on CHB liver paraffin sections}

A small number of patients (10.4\%) underwent liver biopsy for histopathological analysis. Sirius Red staining is a common histochemical method for the detection of liver fibrosis. The Sirius Red staining materials used included: Solution A: $0.5 \mathrm{~g}$ Sirius Red F3B in $500 \mathrm{~mL}$ saturated picric acid; Solution B: $5 \mathrm{~mL}$ acetic acid in $1 \mathrm{~L}$ of distilled water. The staining procedure was carried out per standard methods. Briefly, the sample was warmed to room temperature for $5 \mathrm{~m}$. The paraffin sections were dewaxed, hydrated, and soaked in $100 \%$ alcohol for $10 \mathrm{~m}$. The sections were then washed twice in phosphate-buffered saline, stained in Solution A for $1 \mathrm{~h}$, washed twice in Solution B, and examined under a light microscope to assess fibrosis.

\section{Statistical analyses}

We used SPSS 19.0 for statistical analysis (IBM Corp., Armonk, NY, USA). Means \pm standard deviations $(x \pm s)$ were used for normal distribution measurements, while nonnormal distribution measurement data were expressed as median \pm standard deviation $\left(25^{\text {th }}\right.$ and $75^{\text {th }}$ percentile). The mean values between two groups were compared by $t$-tests or by non-parametric methods if the data was not normally distributed. Count data was analyzed using the chi-square test. $P$ values less than 0.05 were considered statistically significant. For correlation studies, we carried out Pearson's statistical analyses. We classified absolute correlation values as follows: very weak, 0.00-0.19; weak, 0.20-0.39; moderate, 0.40-0.59; strong, 0.60-0.79; and very strong, 0.80-1.00.

\section{Ethics}

The authors declare that this study fully complied with all relevant ethical standards. Informed consent was acquired from all of the patients. This study was approved by the Human Ethics Committee of Shunde Hospital, Southern Medical University, according to the Declaration of Helsinki.

\section{Results}

\section{Clinical features}

Fibrosis is the earliest pathological sign of $\mathrm{CHB}$, and cirrhosis represents the last stage of fibrosis, at which point liver transplantation may be required. In our study, patients were divided into three groups. Group 1 was divided by the degree of fibrosis, consisting of group 1a (S1) with non-significant fibrosis, and group 1b (S2-4) with significant fibrosis. Group 1a (S1) consisted of 1082 male patients (72.8\%) and 404 female patients $(27.2 \%)$, with an average age of $40.0 \pm 10.5$ years. Group $1 \mathrm{~b}$ (S2-4) contained of 618 male patients (79.2\%) and 162 female patients $(20.8 \%)$, with an average age of $44.8 \pm 12.1$ years. Group 2 was stratified by the level of cirrhosis. Group $2 a$ (S1-3) was the non-cirrhosis group, with an average age of $41.0 \pm 10.9$ years and composed of 1540 male patients (74.4\%) and 531 female patients (25.6\%). Group $2 \mathrm{~b}$ (S4) was the early cirrhosis group (average age of $48.4 \pm 12.8$ years), which included 160 male patients (82.1\%)and 35 female patients (17.9\%). Group 3 was divided into non-fatty liver group (3a) and fatty liver group (3b). The non-fatty liver group had an average age of $42.8 \pm 10.9$ years and contained 659 male patients $(69.2 \%)$ and 294 female patients $(30.8 \%)$. The fatty liver group had 1041 male patients (79.3\%) and 272 female patients (20.7\%), with an average age of $40.1 \pm 11.6$ years. In all three groups, the incidence of fatty liver or liver fibrosis was markedly higher in men than in women $(p<0.05)$. Groups $1 \mathrm{~b}, 2 \mathrm{~b}$, and $3 \mathrm{~b}$ suffered from worse or abnormal liver function, fibrosis parameters, and hepatitis $B$ viral load than groups $1 \mathrm{a}, 2 \mathrm{a}$, and $3 \mathrm{a}$ respectively.

In our cohort of 2266 patients, we detected a total of 780 cases with significant liver fibrosis (34.42\%). In men, the incidence of fibrosis detection was higher than in women (36.35\% of men showed fibrosis [618/1700], as opposed to $28.62 \%$ of women [162/566]). This sex difference was statistically significant $(p<0.001)$. All patients were then divided into either a non-significant fibrosis group (further referred to NSF, 1486 patients) or a significant fibrosis group (further referred to as SF, 780 patients). The clinical characteristics of the two groups are shown in Table 1. A comparative 
analysis of the two groups (Table 1) shows age, glutamicpyruvic transaminase (ALT), glutamic-oxaloacetic transaminase (AST), gamma-glutamyltransferase (GGT), albumin (Alb), globin, total bilirubin (TB), direct bilirubin (DB), white blood cell (WBC) count, hemoglobin ( $\mathrm{Hb})$, platelet $(\mathrm{PI})$, blood urea nitrogen, creatinine, uric acid, glucose, total cholesterol, triglycerides, high-density and low-density lipoprotein (HDL and LDL respectively), apolipoprotein $A$ and $B$, hyaluronic acid, laminin, type III and IV procollagen, HBV DNA load, as well as CAP and LSM scores for each patient. Unsurprisingly, most liver function parameters were significantly higher in the SF group than in the NSF group $(p<0.001)$. Blood type III and IV procollagen levels, which represent liver fibrosis severity and HBV DNA load, were furthermore significantly higher in the SF group than in the NSF group $(p<0.001)$. There were no significant differences in age, kidney function, levels of triglycerides, $\mathrm{HDL}$, or apolipoprotein $\mathrm{A}$ between the two groups ( $p>0.05)$.CAP scores were 251.2 \pm 54.3 $\mathrm{db} / \mathrm{m}$ and $251.4 \pm 59.6 \mathrm{db} / \mathrm{m}$ for the NSF group and SF group respectively, indicating no significant difference in CAP values between the two groups $(p>0.05)$. However, there was a significant difference in LSM values $(p<0.001)$; the SF group had a significantly increased LSM value $(15.8 \pm 12.8 \mathrm{kPa})$ compared to the NSF group $(5.0 \pm 1.1 \mathrm{kPa})$.

In total, 195 cases of early cirrhosis were detected among the 2266 patients, representing a rate of $8.61 \%$. Early cirrhosis was more frequently found in men compared to women $(9.41 \%$ of men [160/1700] compared to $6.18 \%$ of women [35/56]). Similar to fibrosis, this sex-specific difference in incidence was statistically significant $(p<0.001)$. Patients were then divided into the non-cirrhosis group (further referred to as NC group, 2071 patients) and early cirrhosis group (further referred to as EC group, 195 patients), according to presence or absence of cirrhosis. The clinical characteristics and comparative analysis of the two groups can be found in Table 2. Most liver function parameters in the EC group were higher than in the NC group $(p<0.001)$. Blood type III and IV procollagen levels, which

Table 1. Clinical features of patients with non-significant fibrosis (S1 group) and significant fibrosis (S2-4 group)

\begin{tabular}{|c|c|c|c|}
\hline & $\begin{array}{l}S 1 \\
n=1486\end{array}$ & $\begin{array}{l}S 2-4 \\
n=780\end{array}$ & $p$ value \\
\hline Male, $n(\%)$ & $1082(68.4)$ & $618(79.2)$ & $<0.001$ \\
\hline Female, $n(\%)$ & $404(31.6)$ & $162(20.8)$ & $<0.001$ \\
\hline Age in years & $40.0 \pm 10.5$ & $44.8 \pm 12.1$ & 0.942 \\
\hline Glutamic-pyruvic transaminase in $\mathrm{U} / \mathrm{L}$ & $32(22,49)$ & $53(31,111)$ & $<0.001$ \\
\hline Glutamic-oxaloacetic transaminase in $\mathrm{U} / \mathrm{L}$ & $30(23,39)$ & $46(32,81)$ & $<0.001$ \\
\hline Gamma-glutamyltransaminase in $\mathrm{U} / \mathrm{L}$ & $23(15,35)$ & $45(26,83)$ & $<0.001$ \\
\hline Albumin in $\mathrm{G} / \mathrm{L}$ & $51.0 \pm 33.0$ & $45.5 \pm 15.4$ & $<0.001$ \\
\hline Globin in G/L & $29.6 \pm 11.2$ & $32.0 \pm 20.2$ & 0.003 \\
\hline Total bilirubin in $\mu \mathrm{mol} / \mathrm{L}$ & $13.9(10.9,17.2)$ & $15.7(12.0,21.1)$ & $<0.001$ \\
\hline Direct bilirubin in $\mu \mathrm{mol} / \mathrm{L}$ & $3.3(2.5,4.4)$ & $4.2(3.0,6.2)$ & $<0.001$ \\
\hline White blood cells in $\mathrm{G} / \mathrm{L}$ & $7.0 \pm 3.3$ & $6.4 \pm 2.2$ & $<0.001$ \\
\hline Hemoglobin in $\mathrm{G} / \mathrm{L}$ & $147.5 \pm 20.7$ & $142.8 \pm 20.3$ & $<0.001$ \\
\hline Platelets in $\mathrm{G} / \mathrm{L}$ & $218.4 \pm 60.2$ & $171.2 \pm 69.0$ & $<0.001$ \\
\hline Blood urea nitrogen in $\mu \mathrm{mol} / \mathrm{L}$ & $5.1 \pm 2.8$ & $5.2 \pm 3.1$ & 0.723 \\
\hline Creatinine in $\mu \mathrm{mol} / \mathrm{L}$ & $81.8 \pm 32.6$ & $84.5 \pm 62.6$ & 0.199 \\
\hline Uric acid in $\mu \mathrm{mol} / \mathrm{L}$ & $397.0 \pm 118.9$ & $389.2 \pm 117.4$ & 0.173 \\
\hline Glucose in $\mathrm{mmol} / \mathrm{L}$ & $7.2 \pm 25.2$ & $7.6 \pm 28.7$ & 0.727 \\
\hline Total cholesterol in $\mathrm{mmol} / \mathrm{L}$ & $4.9(4.4,5.7)$ & $4.7(4.1,5.5)$ & $<0.001$ \\
\hline Triglycerides in $\mathrm{mmol} / \mathrm{L}$ & $1.1(0.8,1.6)$ & $1.0(0.8,1.5)$ & 0.054 \\
\hline High-density lipoprotein in $\mathrm{mmol} / \mathrm{L}$ & $1.3 \pm 0.4$ & $1.3 \pm 0.7$ & 0.552 \\
\hline Low-density lipoprotein in $\mathrm{mmol} / \mathrm{L}$ & $3.1(2.5,3.7)$ & $2.8(2.2,3.5)$ & $<0.001$ \\
\hline Apolipoprotein A in $\mathrm{mmol} / \mathrm{L}$ & $1.3 \pm 0.3$ & $1.3 \pm 0.9$ & 0.830 \\
\hline Apolipoprotein B in $\mathrm{mmol} / \mathrm{L}$ & $1.1 \pm 0.4$ & $1.0 \pm 0.5$ & 0.014 \\
\hline Hyaluronic acid & $51.7(28.7,79.5)$ & $88.5(49.8,160.4)$ & $<0.001$ \\
\hline Laminin & $35.1(21.7,49.8)$ & $48.5(30.5,78.7)$ & $<0.001$ \\
\hline Type III procollagen & $9.2(7.3,11.9)$ & $12.1(9.1,17.5)$ & $<0.001$ \\
\hline Type IV procollagen & $30.6(23.3,39.7)$ & $53.7(34.7,98.8)$ & $<0.001$ \\
\hline HBV DNA load as log10 IU/mL & $3.4 \pm 2.1$ & $4.1 \pm 2.2$ & $<0.001$ \\
\hline Controlled attenuation parameter & $251.2 \pm 54.3$ & $251.4 \pm 59.6$ & 0.942 \\
\hline Liver stiffness measurement & $5.0 \pm 1.1$ & $15.8 \pm 12.8$ & $<0.001$ \\
\hline
\end{tabular}


represent liver fibrosis severity and HBV DNA load, were higher in the EC group than in the NC group $(p<0.001)$ but no significant differences were found in kidney function, triglycerides, HDL and apolipoprotein B between the two groups $(p>0.05)$. Both CAP and LSM values were significantly different between the two groups $(p<0.001$; NC: CAP of $237.4 \pm 57.9$, LSM of 32.7 $\pm 16.0 \mathrm{kPa}$; EC: CAP of $252.6 \pm 55.9$, LSM of $6.5 \pm 2.8 \mathrm{kPa}$ ).

In total, 1313 fatty liver cases were detected among the 2266 patients, marking an overall incidence of 57.94\%. Again, incidence was higher in males than in females, with $61.24 \%$ (1041/1700) of men showing fatty liver compared to $48.06 \%$ of women $(272 / 566)$. The difference in incidence between the two sexes was statistically significant $(p<0.001)$. Patients were divided into a non-fatty liver group (NFL, 953 patients) and fatty liver group (FL, 1313 patients). The clinical characteristics and comparative analysis of the two groups are shown in Table 3. Blood glucose levels, total cholesterol, triglycerides, $\mathrm{LDL}$, apolipoprotein $\mathrm{B}$, laminin and type III procollagen were significantly higher in NFL patients compared to FL patients $(p<0.05)$. On the other hand, blood HDL, hyaluronic acid and type IV procollagen levels and HBV DNA load were significantly lower in the NFL group than in the FL group $(p<0.05)$. Here, we found significant differences in the CAP score between the groups $(p<0.001)$. NFL CAP scores were $284(265,313) \mathrm{db} /$ $\mathrm{m}$, while FL CAP scores were $203(182,220) \mathrm{db} / \mathrm{m}$. LSM values were $5.9(4.6,8.3) \mathrm{kPa}$ and $6.0(4.6,9.3) \mathrm{kPa}$ for NFL and FL respectively. There were no significant differences in LSM values between the two groups $(p>0.05)$.

\section{Sirius Red staining of liver paraffin sections}

We obtained liver sections from $10.4 \%$ of the patients (235/ 2266) who underwent biopsy. Fig. 1 shows representative pictures from the Sirius Red staining of liver sections at different stages of liver fibrosis, indicating that the staining matched the FibroScan results very well.

Table 2. Clinical features of patients with non-cirrhosis (S1-3 group) and early cirrhosis (S4 group)

\begin{tabular}{|c|c|c|c|}
\hline & $\begin{array}{l}S 1-3 \\
n=2071\end{array}$ & $\begin{array}{l}\text { S4 } \\
n=195\end{array}$ & $p$ value \\
\hline Male, $n(\%)$ & $1540(74.4)$ & $160(82.1)$ & 0.018 \\
\hline Female, $n(\%)$ & $531(25.6)$ & 35 (17.9) & $<0.001$ \\
\hline Age in years & $41.0 \pm 10.9$ & $48.4 \pm 12.8$ & $<0.001$ \\
\hline Glutamic-pyruvic transaminase in $\mathrm{U} / \mathrm{L}$ & $35(23,60)$ & $66(36,196)$ & $<0.001$ \\
\hline Glutamic-oxaloacetic transaminase in $\mathrm{U} / \mathrm{L}$ & $32(24,45)$ & $66(45,158)$ & $<0.001$ \\
\hline Gamma-glutamyltransaminase in $U / L$ & $26(17,43)$ & $86(51,166)$ & $<0.001$ \\
\hline Albumin in $\mathrm{G} / \mathrm{L}$ & $49.9 \pm 29.3$ & $40.0 \pm 9.9$ & $<0.001$ \\
\hline Globin in $\mathrm{G} / \mathrm{L}$ & $30.1 \pm 13.9$ & $34.3 \pm 23.2$ & 0.015 \\
\hline Total bilirubin in $\mu \mathrm{mol} / \mathrm{L}$ & $14.1(11.0,17.7)$ & $22.3(16.4,30.9)$ & $<0.001$ \\
\hline Direct bilirubin in $\mu \mathrm{mol} / \mathrm{L}$ & $3.5(2.6,4.7)$ & $6.7(4.6,12.3)$ & $<0.001$ \\
\hline White blood cells in G/L & $6.9 \pm 3.1$ & $5.6 \pm 2.0$ & $<0.001$ \\
\hline Hemoglobin in $\mathrm{G} / \mathrm{L}$ & $147.3 \pm 19.5$ & $131.6 \pm 25.7$ & $<0.001$ \\
\hline Platelets in G/L & $209.3 \pm 36.1$ & $127.6 \pm 63.1$ & $<0.001$ \\
\hline Blood urea nitrogen in $\mu \mathrm{mol} / \mathrm{L}$ & $5.2 \pm 2.9$ & $5.3 \pm 3.6$ & 0.621 \\
\hline Creatinine in $\mu \mathrm{mol} / \mathrm{L}$ & $82.2 \pm 33.7$ & $88.8 \pm 110.8$ & 0.457 \\
\hline Uric acid in $\mu \mathrm{mol} / \mathrm{L}$ & $395.6 \pm 118.1$ & $380.1 \pm 122.8$ & 0.140 \\
\hline Glucose in $\mathrm{mmol} / \mathrm{L}$ & $5.7(5.3,6.1)$ & $5.6(5.1,6.6)$ & 0.709 \\
\hline Total cholesterol in $\mathrm{mmol} / \mathrm{L}$ & $4.9(4.3,5.6)$ & $4.6(3.9,5.2)$ & $<0.001$ \\
\hline Triglycerides in $\mathrm{mmol} / \mathrm{L}$ & $1.1(0.8,1.6)$ & $1.0(0.8,1.6)$ & 0.054 \\
\hline High-density lipoprotein in $\mathrm{mmol} / \mathrm{L}$ & $1.3 \pm 0.5$ & $1.3 \pm 0.4$ & 0.074 \\
\hline Low-density lipoprotein in $\mathrm{mmol} / \mathrm{L}$ & $3.0(2.5,3.6)$ & $2.5(1.9,3.0)$ & $<0.001$ \\
\hline Apolipoprotein A in $\mathrm{mmol} / \mathrm{L}$ & $1.4 \pm 0.7$ & $1.1 \pm 0.4$ & 0.017 \\
\hline Apolipoprotein B in mmol/L & $1.1 \pm 0.5$ & $1.0 \pm 0.3$ & 0.184 \\
\hline Hyaluronic acid & $56.1(32.9,88.8)$ & $213.0(114.7,411.5)$ & $<0.001$ \\
\hline Laminin & $36.4(23.7,53.3)$ & $80.5(55.9,140.4)$ & $<0.001$ \\
\hline Type III procollagen & $9.6(7.5,12.8)$ & $17.5(13.4,24.2)$ & $<0.001$ \\
\hline Type IV procollagen & $33.3(25.0,47.4)$ & $146.6(80.3,244.7)$ & $<0.001$ \\
\hline HBV DNA load as log10 IU/mL & $3.6 \pm 2.1$ & $4.5 \pm 2.4$ & $<0.001$ \\
\hline Controlled attenuation parameter & $252.6 \pm 55.9$ & $237.4 \pm 57.9$ & $<0.001$ \\
\hline Liver stiffness measurement & $6.5 \pm 2.8$ & $32.7 \pm 16.0$ & $<0.001$ \\
\hline
\end{tabular}


He T. et al: FibroScan for $\mathrm{CHB}$

Table 3. Clinical features of patients with non-fatty liver and fatty liver

\begin{tabular}{|c|c|c|c|}
\hline & $\begin{array}{l}\text { Non-fatty liver } \\
n=953\end{array}$ & $\begin{array}{l}\text { Fatty liver } \\
n=1313\end{array}$ & $p$ value \\
\hline Male, $n(\%)$ & $659(69.2)$ & $1041(79.3)$ & $<0.001$ \\
\hline Female, $n(\%)$ & $294(30.8)$ & $272(20.7)$ & $<0.001$ \\
\hline Age in years & $42.8 \pm 10.9$ & $40.1 \pm 11.6$ & $<0.001$ \\
\hline Glutamic-pyruvic transaminase in $U / L$ & $38(25,61)$ & $34(22,68)$ & 0.033 \\
\hline Glutamic-oxaloacetic transaminase in $\mathrm{U} / \mathrm{L}$ & $31(23,44)$ & $37(27,59)$ & $<0.001$ \\
\hline Gamma-glutamyltransaminase in $U / L$ & $31(20,51)$ & $22(14,44)$ & $<0.001$ \\
\hline Albumin in $\mathrm{G} / \mathrm{L}$ & $50.6 \pm 34.3$ & $46.9 \pm 16.6$ & 0.003 \\
\hline Globin in $\mathrm{G} / \mathrm{L}$ & $29.5 \pm 9.3$ & $31.7 \pm 20.3$ & 0.004 \\
\hline Total bilirubin in $\mu \mathrm{mol} / \mathrm{L}$ & $14.0(10.8,17.5)$ & $15.3(11.9,20.0)$ & $<0.001$ \\
\hline Direct bilirubin in $\mu \mathrm{mol} / \mathrm{L}$ & $3.4(2.5,4.6)$ & $3.9(2.8,5.4)$ & $<0.001$ \\
\hline White blood cells in $\mathrm{G} / \mathrm{L}$ & $7.2 \pm 3.6$ & $6.2 \pm 1.8$ & $<0.001$ \\
\hline Hemoglobin in $\mathrm{G} / \mathrm{L}$ & $148.5 \pm 20.4$ & $142.1 \pm 20.4$ & $<0.001$ \\
\hline Platelets in G/L & $212.3 \pm 65.9$ & $187.8 \pm 66.3$ & $<0.001$ \\
\hline Blood urea nitrogen in $\mu \mathrm{mol} / \mathrm{L}$ & $5.3 \pm 3.4$ & $5.0 \pm 2.2$ & 0.032 \\
\hline Creatinine in $\mu \mathrm{mol} / \mathrm{L}$ & $82.3 \pm 26.4$ & $83.2 \pm 62.5$ & 0.617 \\
\hline Uric acid in $\mu \mathrm{mol} / \mathrm{L}$ & $414.2 \pm 119.0$ & $365.8 \pm 116.7$ & $<0.001$ \\
\hline Glucose in $\mathrm{mmol} / \mathrm{L}$ & $5.8(5.4,6.3)$ & $5.5(5.2,5.9)$ & $<0.001$ \\
\hline Total cholesterol in $\mathrm{mmol} / \mathrm{L}$ & $5.1(4.4,5.8)$ & $4.7(4.0,5.4)$ & $<0.001$ \\
\hline Triglycerides in $\mathrm{mmol} / \mathrm{L}$ & $1.3(0.9,1.8)$ & $0.9(0.7,1.2)$ & $<0.001$ \\
\hline High-density lipoprotein in $\mathrm{mmol} / \mathrm{L}$ & $1.3 \pm 0.4$ & $1.4 \pm 0.7$ & $<0.001$ \\
\hline Low-density lipoprotein in $\mathrm{mmol} / \mathrm{L}$ & $3.2(2.6,3.8)$ & $2.8(2.3,3.3)$ & $<0.001$ \\
\hline Apolipoprotein A in $\mathrm{mmol} / \mathrm{L}$ & $1.3 \pm 0.8$ & $1.3 \pm 0.4$ & 0.791 \\
\hline Apolipoprotein $B$ in $\mathrm{mmol} / \mathrm{L}$ & $1.1 \pm 0.5$ & $1.0 \pm 0.3$ & $<0.001$ \\
\hline Hyaluronic acid & $56.1(32.0,98.7)$ & $64.3(38.9,101.3)$ & 0.038 \\
\hline Laminin & $65.1(21.1,52.0)$ & $44.1(28.4,66.5)$ & $<0.001$ \\
\hline Type III procollagen & $10.3(7.8,14.7)$ & $9.8(7.5,13.5)$ & 0.046 \\
\hline Type IV procollagen & $34.7(25.0,51.7)$ & $35.7(27.0,58.3)$ & 0.032 \\
\hline HBV DNA load as log10 IU/mL & $3.3 \pm 2.0$ & $4.1 \pm 2.2$ & $<0.001$ \\
\hline Controlled attenuation parameter & $284(265,313)$ & $203(182,220)$ & $<0.001$ \\
\hline Liver stiffness measurement & $5.9(4.6,8.3)$ & $6.0(4.6,9.3)$ & 0.119 \\
\hline
\end{tabular}

\section{Statistical analysis results}

Spearman's correlation analyses showed that CAP scores and LSM values correlated with sex $(r=0.137)$, age $(r=0.119)$, ALT $(r=0.082)$, AST $(r=-0.172)$, GGT $(r=0.225)$, Alb $(r=0.150)$, globulin $(r=-0.107)$, TB $(r=-0.132)$, DB $(r=-0.145)$, WBC count $(r=0.254), \mathrm{Hb}(r=0.205), \mathrm{Pl}(r=0.206)$, total cholesterol $(r=0.214)$, HDL $(r=-0.243)$, LDL $(r=0.255)$, apolipoprotein B $(r=0.217)$, hyaluronic acid $(r=-0.069)$, laminin $(r=-0.188)$, procollagen type IV $(r=-0.067)$, and hepatitis B viral DNA load $(r=-0.216)$.

\section{Discussion}

$\mathrm{CHB}$ is a chronic hepatophagocytic viral infection of the human liver, which induces deleterious protracted immune responses and ultimately leads to progressive liver fibrosis or cirrhosis as well as a greatly increased risk for hepatocellular carcinoma. ${ }^{12,13}$ Accurately determining the staging and severity of $\mathrm{CHB}$ is therefore extremely important for selecting the appropriate therapeutic interventions. Currently, such estimations are made largely based on serial serological biochemical tests that are intended to reflect liver injury and repair responses in patients, but these tests are limited in their ability to fully reflect the entirety of liver function in CHB patients. ${ }^{14,15}$ Liver biopsies followed by pathological staining-including Sirius Red staining, Masson's Trichrome staining, and Oil Red O staining-are commonly used for diagnosis of liver fibrosis and hepatic steatosis. However, these methods are limited as screening tools in medical practice because of the invasive nature of the biopsies. ${ }^{16}$

FibroScan is already used worldwide in the pathological analysis of various liver diseases, including CHB and NFALD. The LSM and CAP values measured by FibroScan were previously found to accurately reflect liver steatosis and fibrosis. ${ }^{17-19}$ However, a larger amount of $\mathrm{CHB}$ patient 

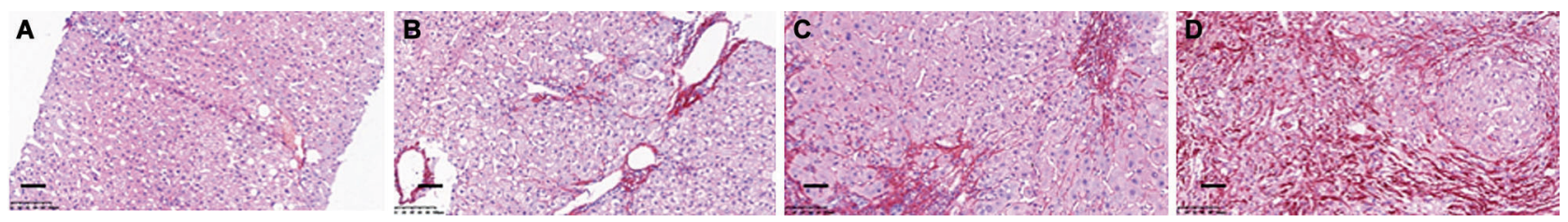

Fig. 1. Comparison of Sirius Red staining with LSM values on CHB liver tissues. (A) Normal control, LSM <7.0 kPa. (B) Significant fibrosis, LSM:7.0 kPa-9.5 kPa. (C) Severe fibrosis, LSM: $9.5 \mathrm{kPa}-12.5 \mathrm{kPa}$. (D) Cirrhosis, LSM>12.0 kPa. Scale bar: $100 \mu \mathrm{m}$.

Abbreviations: CHB, chronic hepatitis B; LSM, liver stiffness measurement.

FibroScan data was required to fully assess its utility in detecting human fatty liver or liver fibrosis diseases. In the current study, we found that the two FibroScan measurements, LSM and CAP, taken from $\mathrm{CHB}$ patients' livers were weakly or very weakly correlated to blood chemical test results, indicating that both LSM and CAP are independent indicators of disease. LSM values were furthermore found to be closely associated with the results of fibrosis staining. Our findings are consistent with several previous studies. Christiansen et al. ${ }^{20}$ conducted a 5 -year prospective study for CHB and hepatitis $\mathrm{C}$ patients. They found that patients with LSM values $\geq 17 \mathrm{kPa}$ have an increased risk of liver diseases, suggesting that a single LSM value cannot be used alone to make clinical decisions.

After analysis of 307 patients who underwent liver biopsy and LSM determination, Li et al. ${ }^{21}$ found that LSM was an independent indicator for different stages of fibrosis $(p<0.001)$. LSM values combined with biochemical indices showed potential value for assessment of CHB-related liver fibrosis.

Performing a multivariate analyses, Zhang et al. ${ }^{22}$ showed that the cumulative probability of hepatocellular carcinoma development in patients with lower liver stiffness was significantly lower compared to patients with increased liver stiffness $(p<0.05)$.

Fatty liver is caused by excessive accumulation of fat in hepatocytes but its exact pathogenesis is still unclear. Similarly, the pathomechanisms underlying fibrosis remain to be elucidated. Eddowes et al. ${ }^{11}$ collected data from 450 suspected NAFLD patients at seven medical centers in the UK and found that CAP scores and LSM values determined by FibroScan were an efficient non-invasive means to assess hepatic steatosis and fibrosis respectively.

In conclusion, our study as well as previous work have shown that FibroScan is a rapid and non-invasive method for detection of fatty liver and liver fibrosis. Its results are not strongly correlated to blood chemistry tests, and may therefore be used to independently assess the presence and development of fatty liver or liver fibrosis.

\section{Funding}

Medical Scientific Research Foundation of Guangdong Province of China (No: A2016450 and A2013695).

\section{Conflict of interest}

The authors have no conflict of interests related to this publication.

\section{Author contributions}

Study design (JD), data collection ( $\mathrm{TH}, \mathrm{JL}, \mathrm{YO})$, data analysis $(\mathrm{JD}, \mathrm{ZZ})$, pathological staining $(\mathrm{GL}, \mathrm{XC})$, statistical analysis $(\mathrm{ZZ})$, and drafting the article (JD).

\section{References}

[1] Hiroi K, Matsusaki T, Kaku R, Umeda Y, Yagi T, Morimatsu H. Postoperative course of serum albumin levels and organ dysfunction after liver transplantation. Transplant Proc 2019;51:2750-2754. doi: 10.1016/j.transproceed. 2019.01.199.

[2] Grabherr F, Grander C, Effenberger M, Adolph TE, Tilg H. Gut dysfunction and non-alcoholic fatty liver disease. Front Endocrinol (Lausanne) 2019;10:611. doi: 10.3389 /fendo.2019.00611.

[3] Cerletti C, Colucci M, Storto M, Semeraro F, Ammollo CT, Incampo F, et al. Randomised trial of chronic supplementation with a nutraceutical mixture in subjects with non-alcoholic fatty liver disease. $\mathrm{Br}$ ] Nutr 2020;123:190-197. doi: $10.1017 /$ S0007114519002484.

[4] Sun J, Li Y, Sun X, Liu Y, Zheng D, Fan L. Association between abdominal obesity and liver steatosis and fibrosis among patients with chronic hepatitis B measured by Fibroscan. Exp Ther Med 2019;18:1891-1898. doi: 10. 3892/etm.2019.7727.

[5] Shen F, Mi YQ, Xu L, Liu YG, Wang XY, Pan Q, et al. Moderate to severe hepatic steatosis leads to overestimation of liver stiffness measurement in chronic hepatitis B patients without significant fibrosis. Aliment Pharmacol Ther 2019;50:93-102. doi: 10.1111/apt.15298.

[6] Sasso M, Miette V, Sandrin L, Beaugrand M. The controlled attenuation parameter (CAP): a novel tool for the non-invasive evaluation of steatosis using Fibroscan. Clin Res Hepatol Gastroenterol 2012;36:13-20. doi: 10. 1016/j.clinre.2011.08.001.

[7] Hansen JF, Christiansen KM, Staugaard B, Moessner BK, Lillevang S, Krag A et al. Combining liver stiffness with hyaluronic acid provides superior prognostic performance in chronic hepatitis C. PLoS One 2019;14:e0212036. doi: 10.1371/journal.pone.0212036.

[8] Hasan EM, Abd Al Aziz RA, Sabry D, Darweesh SK, Badary HA, Elsharkawy A et al. Genetic Variants in nicotinamide-N-methyltransferase (NNMT) gene are related to the stage of non-alcoholic fatty liver disease diagnosed by controlled attenuation parameter (CAP)-fibroscan. J Gastrointestin Liver Dis 2018;27:265-272. doi: 10.15403/jgld.2014.1121.273.wsh.

[9] Hartl J, Ehlken H, Sebode M, Peiseler M, Krech T, Zenouzi R, et al. Usefulness of biochemical remission and transient elastography in monitoring disease course in autoimmune hepatitis. J Hepatol 2018;68:754-763. doi: 10. 1016/j.jhep.2017.11.020.

[10] Armas Cayarga A, Perea Hernández Y, González González YJ, Figueredo Lago JE, Valdivia Álvarez IY, Gómez Cordero I, et al. Performance characteristics of a fast real-time PCR assay for hepatitis $B$ virus DNA quantification. Biologicals 2019;58:22-27. doi: 10.1016/j.biologicals.2019.01.003.

[11] Eddowes PJ, Sasso M, Allison M, Tsochatzis E, Anstee QM, Sheridan D, et al. Accuracy of FibroScan controlled attenuation parameter and liver stiffness measurement in assessing steatosis and Fibrosis in patients with nonalcoholic fatty liver disease. Gastroenterology 2019;156:1717-1730. doi: 10 . 1053/j.gastro.2019.01.042

[12] Weinberger B, Haks MC, de Paus RA, Ottenhoff THM, Bauer T, Grubeck-Loebenstein $B$. Impaired immune response to primary but not to booster vaccination against hepatitis B in older adults. Front Immunol 2018;9:1035. doi: 10.3389/fimmu.2018.01035.

[13] Nitschke $K$, Luxenburger $H$, Kiraithe MM, Thimme R, Neumann-haefelin C. CD8+ T-cell responses in hepatitis B and C: The (HLA-) A, B, and C of hepatitis $B$ and $C$. Dig Dis 2016;34:396-409. doi: 10.1159/000444555.

[14] Portilho MM, Mendonça ACDF, Bezerra CS, do Espirito-Santo MP, de Paula VS, Nabuco LC, et al. Usefulness of in-house real time PCR for HBV DNA quantification in serum and oral fluid samples. J Virol Methods 2018;256:100-106. doi: $10.1016 /$ j.jviromet.2018.03.001

[15] Kim H, Hur M, Bae E, Lee KA, Lee WI. Performance evaluation of cobas HBV real-time PCR assay on Roche cobas 4800 System in comparison with COBAS AmpliPrep/COBAS TaqMan HBV Test. Clin Chem Lab Med 2018;56:11331139. doi: $10.1515 / \mathrm{cclm}-2017-1133$.

[16] Scoazec JY. Liver biopsy: Which role in patient management? Ann Pathol 2010;30:464-469. doi: 10.1016/j.annpat.2010.08.026. 
He T. et al: FibroScan for $\mathrm{CHB}$

[17] Xu XY, Wang WS, Zhang QM, Li JL, Sun JB, Qin TT, et al. Performance of common imaging techniques vs serum biomarkers in assessing fibrosis in patients with chronic hepatitis B: A systematic review and meta-analysis. World J Clin Cases 2019;7:2022-2037. doi: 10.12998/wjcc.v7.i15.2022.

[18] Parikh P, Ryan JD, Tsochatzis EA. Fibrosis assessment in patients with chronic hepatitis B virus (HBV) infection. Ann Transl Med 2017;5:40. doi: 10. 21037/atm.2017.01.28.

[19] Afdhal NH, Bacon BR, Patel K, Lawitz EJ, Gordon SC, Nelson DR, et al. Accuracy of fibroscan, compared with histology, in analysis of liver fibrosis in patients with hepatitis B or C: a United States multicenter study. Clin Gastroenterol Hepatol 2015;13:772-779.e1-3. doi: 10.1016/j.cgh.2014.12.014.
[20] Christiansen KM, Mössner BK, Hansen JF, Jarnbjer EF, Pedersen C, Christensen $\mathrm{PB}$. Liver stiffness measurement among patients with chronic hepatitis $B$ and C: results from a 5-year prospective study. PLoS One 2014;9:e111912. doi: 10.1371/journal.pone.0111912.

[21] Li B, Zhang L, Zhang Z, Yan G, Zhu L, Lu W, et al. A noninvasive indicator for the diagnosis of early hepatitis $B$ virus-related liver fibrosis. Eur J Gastroenterol Hepatol 2019;31:218-223. doi: 10.1097/MEG.0000000000001281.

[22] Zhang Y, Wang C, Li H, Ding Y. Decreased liver stiffness by transient elastography indicates lower incidence of hepatocellular carcinoma in patients with chronic hepatitis B. Medicine (Baltimore) 2019;98:e13929. doi: 10. 1097/MD.0000000000013929. 